\title{
APLICAÇÃO DO MRP II: ESTUDO DE CASO EM UMA EMPRESA DE INJETÁVEIS LOCALIZADA NA CIDADE DE RUSSAS - CEARÁ
}

\author{
Rayane Araújo Lima (Universidade Federal do Ceará) rayanearaujo@alu.ufc.br \\ Hévilla Souza Oliveira (Universidade Federal do Ceará) hevillasouza@alu.ufc.br \\ Carla Amanda Matos Lima (Universidade Federal do Ceará) amandamts1@alu.ufc.br \\ Daiane de Oliveira Costa (Universidade Federal do Ceará) daiane_oliveirac@ hotmail.com \\ Sâmara Margarida Santana Soares (Universidade Potiguar) smargarida-@hotmail.com
}

\section{Resumo}

Analisando o atual cenário de competitividade no qual as empresas estão inseridas, faz-se necessário por parte destas a busca por ferramentas que possibilitem a comunicação entre os diversos setores da empresa, visando a eliminação de desperdícios e auxílio na tomada de decisões estratégicas para tornar o planejamento da produção mais eficiente e organizado. Nesse contexto, foi proposta a aplicação do MRP II para os principais produtos de uma empresa de injetáveis localizada no interior do Ceará, utilizando a previsão de demanda para os meses de abril e maio de 2019. Com a definição da predição, realizou-se o Planejamento Agregado para a família de produtos e o Planejamento Mestre da Produção. Após estas etapas, foram desenvolvidos os cálculos das necessidades de matéria-prima e as liberações de ordem de reposição requeridas para o cumprimento das atividades produtivas. Durante a elaboração do trabalho, verificou-se também se a capacidade do maquinário e mão-de-obra supriam o planejamento determinado. A finalização do estudo deu-se pela definição do sequenciamento da produção no módulo do Controle de Chão de Fábrica. Neste trabalho pôde-se concluir que a metodologia abordada proporciona à empresa planejar sua produção da melhor forma possível de modo a atender e satisfazer seus clientes, assegurando maiores benefícios para esta.

Palavras-Chaves: MRP II. Análise de capacidade. Empresa de Injetáveis.

\section{Introdução}

Segundo Corrêa e Gianesi (2002), dada a alta concorrência existente no mercado, as organizações precisam atender a diferentes critérios competitivos, como baixo custo, qualidade elevada, lead-times reduzidos, dentre outros. No entanto, ser excelente em todos esses aspectos é uma difícil tarefa, que requer o desenvolvimento de ferramentas que possibilitem a 
comunicação entre os diversos setores da empresa, visando a eliminação de desperdícios e auxílio na tomada de decisões estratégicas para tornar o planejamento da produção mais eficiente e organizado.

Nesse contexto, o Planejamento dos Recursos de Manufatura (Manufacturing Resources Planning - MRP II) se mostra de grande valia, visto que o seu principal objetivo é "permitir o cumprimento dos prazos de entrega dos pedidos dos clientes com mínima formação de estoques, planejando as compras e a produção de itens componentes para que ocorram apenas nos momentos e quantidades necessárias" (CORRÊA, GIANESI, 2002, p.104).

A utilização do MRP II nas indústrias busca a redução de estoques, que permite a liberação de espaço físico e capital de modo que este possa ser reinvestido para aumentar a produção, além de auxiliar na definição de itens a serem comprados e fabricados, bem como na alocação de equipamentos e colaboradores.

A empresa foco deste estudo atua no ramo de produção de injetáveis plásticos, fabricando principalmente saltos e palmilhas para calçados. Segundo o Relatório Setorial Indústria de Calçados publicado pela Abicalçados (2018), o setor calçadista obteve um crescimento de 1,1\% em sua produção durante o ano de 2017, atingindo uma marca de 908,9 milhões de pares produzidos. O progresso neste setor reflete um aumento na demanda do ramo da indústria estudada.

Diante do que foi apresentado, este estudo busca mostrar a aplicação do MRP II em uma indústria de injetáveis localizada no interior do Ceará, com o objetivo de auxiliar o planejamento das atividades de produção, bem como analisar a capacidade produtiva da empresa para atendimento da demanda.

\section{Referencial teórico}

\subsection{Planejamento e controle da produção}

O Planejamento e Controle da Produção (PCP) é uma área da Engenharia de Produção fundamental para o funcionamento das indústrias, sendo suporte para as tomadas de decisão e responsável pelas atividades de planejar e controlar os processos. (SLACK; CHAMBERS; JOHNSTON, 2009). Bonney (2000) afirma que isto ocorre através de uma hierarquia nas diferentes escalas de tempo auxiliando os gestores no controle de suas operações de forma eficaz conforme as medidas de desempenho da empresa. 
Estender et al. (2017) asseguram ainda que quando este planejamento é inexistente ou ocorre de forma incorreta impacta negativamente em diversos aspectos do processo produtivo, uma vez que pode ocasionar transtornos e afetar diretamente a credibilidade que a empresa possui com os seus consumidores.

\subsection{Manufacturing Resource Planning}

Dentre as metodologias empregadas no PCP, o MRP II aborda o princípio do cálculo das necessidades dos recursos de manufatura, ou seja, refere-se ao planejamento dos recursos para possibilitar a produção. Segundo Lustosa et al. (2008), é um sistema no qual ocorre de forma integrada o planejamento das operações produtivas, além da consideração dos estoques durante os cálculos. É aplicado nas indústrias por meio de ferramentas computacionais, permitindo a gestão dos materiais, pessoas e equipamentos utilizados nos processos que garantam o atendimento da demanda (SILVA, 2012).

A integração dos recursos realizada no sistema MRP II é efetuada por meio da implantação de diferentes módulos que possuem uma relação de interdependência e hierarquia, apresentados a seguir.

\subsubsection{Sales and Operations Planning (S\&OP)}

O primeiro módulo do MRP II, o Sales and Operations Planning (Planejamento Agregado de Vendas e Operações - PVO) é realizado nas organizações devido à dificuldade de se prever a demanda para os produtos individualmente, dado que o mix de produção nas indústrias pode variar em grandes quantidades. Diante disso, Peinado e Graeml (2007) afirmam que uma solução viável seria a junção dos modelos existentes em famílias para que estas possam representar as necessidades da produção.

Olhager, Rudberg e Wikner (2001) alegam que o S\&OP é o planejamento organizacional capaz de manter o equilíbrio entre oferta agregada e demanda agregada, ou seja, atender as necessidades do mercado de forma economicamente viável. Ele se divide em planos de produção e de venda, afetando diretamente o estoque, a carteira de pedidos e os requisitos de capacidade.

\subsubsection{Master Production Shedule (MPS)}

A partir da elaboração do PVO, o planejamento individual para cada produto ocorre por meio do Master Production Shedule (Plano Mestre da Produção - PMP), que especifica os itens que serão produzidos e suas respectivas quantidades para determinado período. Fundamentado no 
planejamento estratégico da empresa e trabalhando no horizonte de tempo de médio prazo, o PMP sintetiza todos os insumos que serão necessários e promove as interações de modo a resultar em informações básicas para planejar a produção (ROMANZINI; RIBEIRO, 2017).

\subsubsection{Material Requirements Planning}

O nível subsequente ao PMP trata-se do MRP (Planejamento da Necessidade de Materiais), que consiste na definição de que partes, quando e quanto é preciso de matéria-prima para a programação dos itens componentes do produto analisado. Este método é um importante aliado na gestão de materiais, visto que desmembra os planos agregados em planejamentos específicos, além de possibilitar a elaboração da análise de capacidade (BERTAGLIA, 2009).

Segundo Peinado e Graeml (2007), para a realização e aplicação do MRP se faz necessário conhecer com propriedade especificações detalhadas sobre quais componentes e suas referidas quantidades serão utilizadas na produção de certo item, sendo isto denominado de Estrutura ou Árvore do Produto, conhecido também como BOM (bill of materials). Para a determinação do período indicado para a liberação da ordem de pedido das matérias-primas que permitam o cumprimento dos planos de produção, utiliza-se no MRP matrizes chamadas de Registros Básicos, sendo estes divididos em um número finito de períodos, com linhas que representam as movimentações logísticas e planejamentos (CORRÊA; CORRÊA, 2012).

\subsubsection{Análise de capacidade}

Slack et al. (2006) define capacidade como o nível máximo de atividade de agregação de valor possível de ser realizado em uma determinada operação, dentro de um período, em condições normais de uma indústria. Portanto, a análise de capacidade é uma atividade fundamental para as empresas, pois garante a possibilidade de verificar se a carga de trabalho disponível conseguirá atender à produção demandada.

Ao nível do PVO, esta análise é denominada de Resource Requirements Planning - RRP e tem por objetivo garantir a viabilidade do PVO em termos de capacidade produtiva, contribuindo assim com as decisões tomadas diante de empecilhos (VENAZI e SILVA, 2013). Já no PMP a análise de capacidade é conhecida como Rough-Cut Capacity Planning - RCCP (Análise Grosseira da Capacidade) e é feita de forma mais "grosseira", sem precisão, relacionando a necessidade e a disponibilidade dos recursos (MARTINS e LAUGENI, 2012). 


\subsubsection{Shop Floor Control}

Corrêa e Gianesi (2002, p. 137) afirmam que o Controle do Chão de Fábrica (SFC) "é o responsável pela sequenciação das ordens, por centro de produção, dentro de um período de planejamento e pelo controle da produção propriamente dita, no nível da fábrica”, sendo este o último dos módulos principais do MRP II.

Regras de sequenciamento são utilizadas para a determinação da ordem de produção, definindo prioridades devido um parâmetro específico. De acordo com Venanzi e Silva (2013) estas possuem como objetivo principal maximizar a utilização das instalações existentes à proporção que o atraso na entrega das atividades, o número de tarefas cadastradas no sistema e o tempo de conclusão são deduzidos, proporcionando maiores ganhos para a organização ao fim do processo.

\section{Metodologia}

\subsection{Caracterização da pesquisa}

Para o presente estudo, inicialmente, foi realizada uma pesquisa bibliográfica, proporcionando aos autores um maior conhecimento e contato com o tema estudado, através do levantamento de referências já existentes (SILVA, 2015). O trabalho tem a natureza de uma pesquisa aplicada, caracterizada como um estudo de caso, uma vez que se explorou os dados obtidos, tornando capaz a investigação das informações dentro do contexto atual, possibilitando o estudo da realidade da empresa em evidência.

Os objetivos desta pesquisa são classificados como exploratórios, pois buscam interpretar as informações adquiridas e obter uma maior clareza sobre conceitos dos conteúdos pertinentes, bem como formular hipóteses para estudos subsequentes (GIL, 2002). Quanto à abordagem, caracteriza-se como qualitativa e quantitativa, uma vez que foram utilizados dados numéricos e informações fornecidas pelo proprietário, que possibilitaram a interpretação dos fenômenos ocorridos na empresa.

\subsection{Análise e tratamento dos dados}

Para a realização deste trabalho, inicialmente ocorreu uma visita para coleta de dados referentes ao histórico de demanda de doze semanas do primeiro trimestre do ano de 2019; itens mais produzidos e seus respectivos ciclos de produção; preços de venda; quantidades necessárias 
para fabricação; dentre outros aspectos. Esses dados foram utilizados para propor o MRP II com base na demanda prevista para os meses de abril e maio de 2019.

Dentre os produtos fabricados pela empresa foi escolhida a família de saltos, já que essa possui a maior representatividade dentro da produção, realizando-se a análise do seu processo produtivo. Com base em uma previsão de demanda para os 5 modelos mais solicitados pelos clientes (40210, 40262, 40263, 40305 e 40328), calculou-se o PVO, objetivando um balanceamento entre a demanda prevista e a produção. Além disso, analisou-se através do cálculo de capacidade se a instalação conseguiria atender à demanda.

Logo após os resultados obtidos, o cálculo do PMP proporcionou desagregar a demanda mensal em semanas, bem como o estudo da disponibilidade de recursos (análise do RCCP). Desta forma, a programação da produção foi feita por meio do MRP elaborando-se a árvore do produto e o planejamento detalhado das necessidades de materiais. Por fim, partiu-se para determinação do SFC, no qual realizou-se o sequenciamento da produção para garantir o cumprimento do plano elaborado anteriormente e possibilitar os feedbacks necessários para o andamento da produção, concluindo assim a elaboração do sistema MRP II. A Figura 1 descreve de maneira visual todas as etapas do estudo.

Figura 1 - Etapas para implantação do MRP II

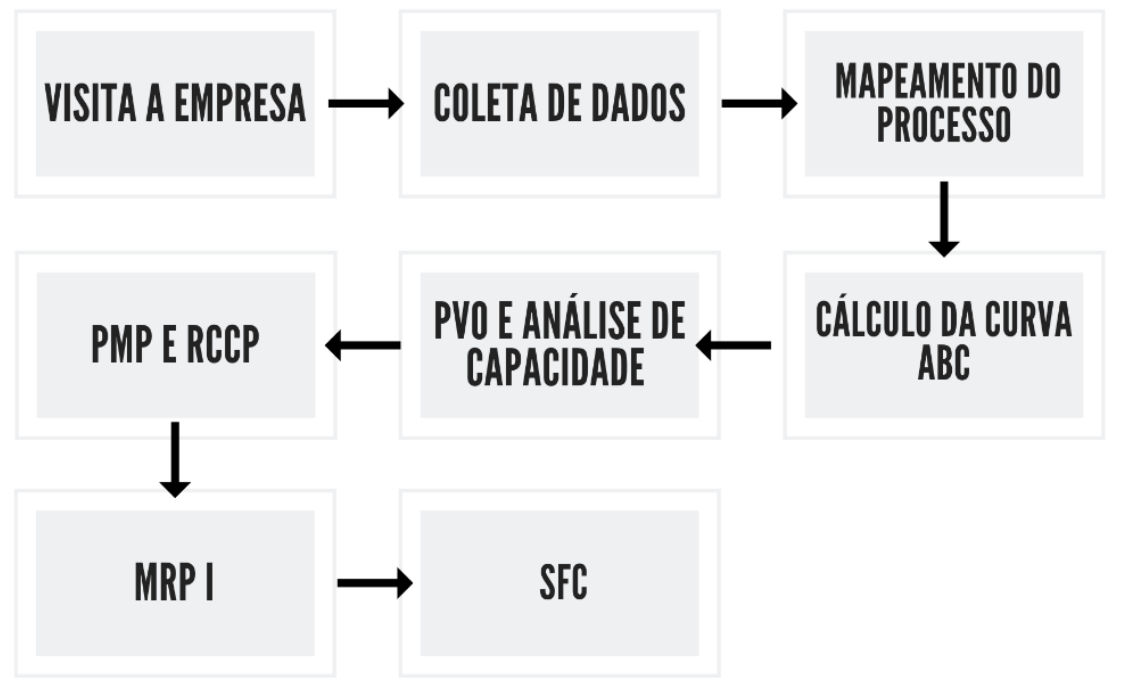

Fonte: Autores (2020) 


\section{Apresentação dos resultados da pesquisa}

\subsection{Caracterização da empresa e do processo produtivo}

Fundada em 1984, a empresa iniciou suas atividades na cidade de Novo Hamburgo, localizada no Rio Grande do Sul. No decorrer dos anos cresceu e ganhou mercado, instalando, no ano de 2008, uma nova unidade fabril em Russas, localizada no interior do Ceará. A planta citada atua produzindo principalmente saltos, anabelas e palmilhas e conta com mais de 200 colaboradores. A produção de bens e serviços ocorre através de um processo de transformação por meio do qual o input, popularmente chamado de ABS, são convertidos em outputs, os saltos. Quanto ao processo de fabricação, foi realizado o fluxograma (Anexo I) considerando os principais modelos da família de saltos. Ao receber o pedido, este é encaminhado ao setor de PCP da empresa, onde ocorre o planejamento diário de como irá proceder a fabricação.

Caso exista estoque de matéria-prima suficiente para produzir os itens, o pedido segue para a linha de produção que funciona durante dois turnos diários, onde é injetado o ABS para a confecção dos saltos. Se não houver matéria-prima, é necessário aguardar a chegada desta para só então continuar com a produção. Após ser injetado, todos os saltos passam por um processo de resfriamento.

Posteriormente faz-se uma avaliação para saber se o modelo em questão necessita passar pela etapa de lixamento. Caso seja necessário, o item será lixado e somente após esta etapa seguirá para o processo de empacotamento. Em seguida, os pedidos serão devidamente despachados após a emissão de nota fiscal.

\subsection{Elaboração do PVO}

Primeiramente, foi necessário realizar a identificação dos principais produtos da organização. Para isso, foram levantadas informações junto ao setor de produção e financeiro, que apontaram estes como sendo 5 modelos pertencentes à família de saltos, assim identificados: 42180, 38445, 17802, 33721, 34284. Estes representam 56\% da família como um todo.

Em seguida, foi elaborada uma previsão de demanda para as semanas referentes aos meses de abril e maio, através do modelo de média móvel ponderada e contando com o histórico de dados da organização, obtendo os resultados apresentados no Quadro 1. 
Quadro 1 - Demanda total dos três meses e volume de produção de cada modelo

\begin{tabular}{|c|c|c|c|c|c|c|c|}
\hline \multicolumn{9}{|c|}{ PREVISÃO SEMANAL } \\
\hline \multicolumn{3}{|c|}{ ABRIL } & \multicolumn{4}{c|}{ MAIO } \\
\hline SEM. 1 & SEM. 2 & SEM. 3 & SEM. 4 & SEM. 1 & SEM. 2 & SEM. 3 & SEM. 4 \\
\hline 16037 & 15744 & 11766 & 14380 & 9670 & 9493 & 7095 & 8671 \\
\hline
\end{tabular}

Fonte: Autores (2020)

\subsubsection{Análise de capacidade do PVO}

Para a análise de capacidade levou-se em consideração a soma dos dados coletados da produção dos três meses, na qual foi possível encontrar a proporção total de fabricação de cada produto frente à família (Quadro 2).

Quadro 2 - Demanda total dos três meses e volume de produção de cada modelo

\begin{tabular}{|c|c|c|}
\hline \multicolumn{3}{|c|}{ CÁLCULO DA PORCENTAGEM - FAMÍLIA } \\
\hline MODELO & DEMANDA & PORCENTAGEM \\
\hline M40210 & 42180 & $25,3 \%$ \\
\hline M40262 & 34284 & $20,6 \%$ \\
\hline M40263 & 17802 & $10,7 \%$ \\
\hline M40305 & 33721 & $20,3 \%$ \\
\hline M40328 & 38445 & $23,1 \%$ \\
\hline
\end{tabular}

Fonte: Autores (2020)

As informações adquiridas de segundos trabalhados a cada turno, o lead time produtivo de cada par de salto e os segundos disponíveis para os meses de abril e maio são mostrados nos Quadros 3,4 e 5 respectivamente.

Quadro 3 - Total de segundos trabalhados por turno

\begin{tabular}{|c|c|c|}
\cline { 2 - 3 } \multicolumn{1}{c|}{} & DIA & NOITE \\
\hline QNTD. DE HORAS TRABALHADAS POR TURNO: & 4,9 & 4,48 \\
\hline QNTD. DE SEGUNDOS TRABALHADAS POR TURNO: & 17740,8 & 16128 \\
\hline
\end{tabular}

Fonte: Autores (2020)

Quadro 4 - Lead time produtivo de cada par de salto em segundos

\begin{tabular}{|c|c|c|c|c|c|}
\hline \multicolumn{7}{|c|}{ PRODUÇÃO PARA CADA PAR DO PRODUTO $(\mathrm{s})$} \\
\hline CENTRO DE TRABALHO & 40210 & 40262 & 40263 & 40305 & 40328 \\
\hline INJEÇÃO & 27,5 & 25 & 30 & 21,6 & 27,5 \\
\hline LIXAMENTO & 14,4 & 0 & 0 & 0 & 0 \\
\hline
\end{tabular}

Fonte: Autores (2020) 
Quadro 5 - Segundos disponíveis nos meses de abril e maio

\begin{tabular}{|c|c|c|}
\cline { 2 - 3 } \multicolumn{1}{c|}{} & \multicolumn{2}{c|}{ SEGUNDOS DISPONIVEIS } \\
\cline { 2 - 3 } \multicolumn{1}{c|}{} & ABRIL & MAIO \\
\hline Injeção & 1209116,2 & 1266693,1 \\
\hline Lixamento & 1266693,1 & 1327011,8 \\
\hline
\end{tabular}

Fonte: Autores (2020)

Para o resultado exibido no Quadro 3 utilizou-se o tempo reservado para a produção da família (56\%), e duas máquinas disponíveis no setor de injeção para a produção dos cinco modelos. No Quadro 5, levou-se em consideração o número de equipamentos ou funcionários por centro de trabalho, a quantidade de dias trabalhados no mês, a quantidade de turnos por setor e uma eficiência de $85 \%$.

A determinação da quantidade de segundos necessários para a produção dos itens da família para cada setor, determinada através dos fatores globais, foi realizada multiplicando-se o tempo de produção de cada modelo por seu respectivo volume, como mostra o Anexo II.

O Quadro 6 apresenta os segundos necessários para a fabricação nos meses previstos, que foram encontrados por meio do produto da demanda mensal e dos fatores calculados.

Quadro 6 - Cálculo dos segundos necessários por mês por setor

\begin{tabular}{|c|c|c|}
\hline \multicolumn{3}{|c|}{ CÁLCULO DAS SEGUNDOS NECESSÁRIOS } \\
\hline CENTRO DE TRABALHO & ABRIL & MAIO \\
\hline INJEÇÃO & 1509352,6 & 910093,4 \\
\hline LIXAMENTO & 211396,7 & 127465,8 \\
\hline
\end{tabular}

Fonte: Autores (2020)

Por fim, tornou-se possível o cálculo da utilização através do quociente entre os segundos necessários e os segundos disponíveis (Quadro 7).

Quadro 7 - Cálculo de utilização mensal nos setores de Injeção e Lixamento

\begin{tabular}{|c|c|c|}
\hline \multicolumn{3}{|c|}{ CÁLCULO DA UTILIZAÇÃO } \\
\hline CENTRO DE TRABALHO & ABRIL & MAIO \\
\hline INJEÇÃO & $125 \%$ & $72 \%$ \\
\hline LIXAMENTO & $17 \%$ & $10 \%$ \\
\hline
\end{tabular}

Fonte: Autores (2020) 
O resultado mostra que, no setor de injeção, a capacidade produtiva da empresa se apresenta inferior à capacidade demandada no mês de abril. Devido a isto, é necessário buscar uma alternativa viável para conseguir atender a demanda, logo, é proposto o uso de mais uma máquina (já presente na organização, mas que atualmente não está sendo utilizada para a família estudada). Com isto, a utilização no mês de abril, no setor de injeção, reduz de $125 \%$ para $83 \%$, sendo possível atender à demanda.

\subsection{Elaboração do PMP}

Partindo da previsão de demanda semanal, o cálculo do PMP iniciou-se com a determinação da proporção média de produção para cada produto (Quadro 8).

Quadro 8 - Proporção por modelo por semana

\begin{tabular}{|c|c|c|c|c|c|}
\cline { 2 - 6 } \multicolumn{1}{c|}{} & \multicolumn{5}{c|}{ PROPORÇÃO POR PRODUTO POR SEMANA } \\
\cline { 2 - 6 } \multicolumn{1}{c|}{} & M40210 & M40328 & M40263 & M40305 & M40262 \\
\hline SEMANA 1 & $25,7 \%$ & $31,4 \%$ & $6,8 \%$ & $9,7 \%$ & $26,3 \%$ \\
\hline SEMANA 2 & $29,2 \%$ & $25,4 \%$ & $8,5 \%$ & $21,6 \%$ & $15,3 \%$ \\
\hline SEMANA 3 & $21,7 \%$ & $20,2 \%$ & $14,3 \%$ & $21,4 \%$ & $22,4 \%$ \\
\hline SEMANA 4 & $24,3 \%$ & $13,9 \%$ & $14,2 \%$ & $30,0 \%$ & $17,5 \%$ \\
\hline MÉDIA & $25,2 \%$ & $22,7 \%$ & $11,0 \%$ & $20,7 \%$ & $20,4 \%$ \\
\hline
\end{tabular}

Fonte: Autores (2020)

A partir disto e com base na demanda prevista para cada semana, calculou-se a quantidade demandada para cada modelo (Quadro 9).

Quadro 9 - Demanda semanal por modelo

\begin{tabular}{|c|c|c|c|c|c|c|c|c|}
\hline & \multicolumn{8}{|c|}{ DEMANDA POR PRODUTO } \\
\hline & \multicolumn{4}{|c|}{ ABRIL } & \multicolumn{4}{|c|}{ MAIO } \\
\hline & SEM. 1 & SEM. 2 & SEM. 3 & SEM. 4 & SEM. 1 & SEM. 2 & SEM. 3 & SEM. 4 \\
\hline M40210 & 4046,56 & 3972,63 & 2968,87 & 3628,45 & 2440,00 & 2395,33 & 1790,26 & 2187,92 \\
\hline M40328 & 3645,12 & 3578,52 & 2674,35 & 3268,49 & 2197,94 & 2157,71 & 1612,65 & 1970,87 \\
\hline M40263 & 1758,06 & 1725,94 & 1289,85 & 1576,41 & 1060,07 & 1040,67 & 777,79 & 950,56 \\
\hline M40305 & 3319,21 & 3258,57 & 2435,23 & 2976,26 & 2001,42 & 1964,79 & 1468,47 & 1794,65 \\
\hline M40262 & 3268,05 & 3208,34 & 2397,70 & 2930,39 & 1970,57 & 1934,50 & 1445,83 & 1766,99 \\
\hline
\end{tabular}

Fonte: Autores (2020)

Em seguida, calculou-se o PMP para cada produto (Quadros 10 ao 14). 
Quadro 10 - PMP para o modelo 40210

\begin{tabular}{|c|c|c|c|c|c|c|c|c|c|}
\hline \multirow{2}{*}{ MODELO 40210 } & \multicolumn{9}{|c|}{ PERIOODOS } \\
\cline { 2 - 10 } & SEM 0 & SEM 1 & SEM 2 & SEM 3 & SEM 4 & SEM 5 & SEM 6 & SEM 7 & SEM 8 \\
\hline PREVISÃO DE DEMANDA & & 4047 & 3973 & 2969 & 3629 & 2440 & 2396 & 1791 & 2188 \\
\hline PEDIDOS EM CARTEIRA & & & & & & & & & \\
\hline DEMANDA & & 4047 & 3973 & 2969 & 3629 & 2440 & 2396 & 1791 & 2188 \\
\hline ESTOQUE DE SEGURANÇA & & 0 & 0 & 0 & 0 & 0 & 0 & 0 & 0 \\
\hline PMP & & 4047 & 3973 & 2969 & 3629 & 2440 & 2396 & 1791 & 2188 \\
\hline
\end{tabular}

Fonte: Autores (2020)

Quadro 11 - PMP para o modelo 40328

\begin{tabular}{|c|c|c|c|c|c|c|c|c|c|}
\hline \multirow{2}{*}{ MODELO 40328 } & \multicolumn{9}{|c|}{ PERÍODOS } \\
\cline { 2 - 10 } & SEM 0 & SEM 1 & SEM 2 & SEM 3 & SEM 4 & SEM 5 & SEM 6 & SEM 7 & SEM 8 \\
\hline PREVISÃO DE DEMANDA & & 3646 & 3579 & 2675 & 3269 & 2198 & 2158 & 1613 & 1971 \\
\hline PEDIDOS EM CARTEIRA & & & & & & & & & \\
\hline DEMANDA & & 3646 & 3579 & 2675 & 3269 & 2198 & 2158 & 1613 & 1971 \\
\hline ESTOQUE DE SEGURANÇA & & 0 & 0 & 0 & 0 & 0 & 0 & 0 & 0 \\
\hline PMP & & 3646 & 3579 & 2675 & 3269 & 2198 & 2158 & 1613 & 1971 \\
\hline
\end{tabular}

Fonte: Autores (2020)

Quadro 12 - PMP para o modelo 40263

\begin{tabular}{|c|c|c|c|c|c|c|c|c|c|}
\hline \multirow{2}{*}{ MODELO 40263 } & \multicolumn{9}{|c|}{ PERIOODOS } \\
\cline { 2 - 11 } & SEM 0 & SEM 1 & SEM 2 & SEM 3 & SEM 4 & SEM 5 & SEM 6 & SEM 7 & SEM 8 \\
\hline PREVISÃO DE DEMANDA & & 1759 & 1726 & 1290 & 1577 & 1061 & 1041 & 778 & 951 \\
\hline PEDIDOS EM CARTEIRA & & & & & & & & & \\
\hline DEMANDA & & 1759 & 1726 & 1290 & 1577 & 1061 & 1041 & 778 & 951 \\
\hline ESTOQUE DE SEGURANÇA & & 0 & 0 & 0 & 0 & 0 & 0 & 0 & 0 \\
\hline PMP & & 1759 & 1726 & 1290 & 1577 & 1061 & 1041 & 778 & 951 \\
\hline
\end{tabular}

Fonte: Autores (2020)

Quadro 13 - PMP para o modelo 40305

\begin{tabular}{|c|c|c|c|c|c|c|c|c|c|}
\hline \multirow{2}{*}{ MODELO 40305 } & \multicolumn{9}{|c|}{ PERIOODOS } \\
\cline { 2 - 10 } & SEM 0 & SEM 1 & SEM 2 & SEM 3 & SEM 4 & SEM 5 & SEM 6 & SEM 7 & SEM 8 \\
\hline PREVISÃO DE DEMANDA & & 3320 & 3259 & 2436 & 2977 & 2002 & 1965 & 1469 & 1795 \\
\hline PEDIDOS EM CARTEIRA & & & & & & & & & \\
\hline DEMANDA & & 3320 & 3259 & 2436 & 2977 & 2002 & 1965 & 1469 & 1795 \\
\hline ESTOQUE DE SEGURANÇA & & 0 & 0 & 0 & 0 & 0 & 0 & 0 & 0 \\
\hline PMP & & 3320 & 3259 & 2436 & 2977 & 2002 & 1965 & 1469 & 1795 \\
\hline
\end{tabular}

Fonte: Autores (2020) 
Quadro 14 - PMP para o modelo 40262

\begin{tabular}{|c|c|c|c|c|c|c|c|c|c|}
\hline \multirow{2}{*}{ MODELO 40262 } & \multicolumn{7}{|c|}{ PERÍODOS } \\
\cline { 2 - 10 } & SEM 0 & SEM 1 & SEM 2 & SEM 3 & SEM 4 & SEM 5 & SEM 6 & SEM 7 & SEM 8 \\
\hline PREVISÃO DE DEMANDA & & 3269 & 3209 & 2398 & 2931 & 1971 & 1935 & 1446 & 1767 \\
\hline PEDIDOS EM CARTEIRA & & & & & & & & & \\
\hline DEMANDA & & 3269 & 3209 & 2398 & 2931 & 1971 & 1935 & 1446 & 1767 \\
\hline ESTOQUE DE SEGURANÇA & & 0 & 0 & 0 & 0 & 0 & 0 & 0 & 0 \\
\hline PMP & & 3269 & 3209 & 2398 & 2931 & 1971 & 1935 & 1446 & 1767 \\
\hline
\end{tabular}

Fonte: Autores (2020)

\subsubsection{Análise de capacidade do PMP}

Para a análise de capacidade do PMP calculou-se o tempo de trabalho por semana através da multiplicação dos valores de segundos diários disponíveis pelos dias úteis por semana, levandose em consideração todos os critérios também utilizados para o cálculo da análise de capacidade ao nível do PVO, os resultados são exibidos no Quadro 15.

Quadro 15 - Segundos disponíveis por semana

\begin{tabular}{|c|c|c|c|c|c|c|c|c|}
\cline { 2 - 9 } \multicolumn{1}{c|}{} & \multicolumn{7}{c|}{ SEGUNDOS DISPONIVEIS } \\
\cline { 2 - 9 } \multicolumn{1}{c|}{} & SEM 1 & SEM 2 & SEM 3 & SEM 4 & SEM 5 & SEM 6 & SEM 7 & SEM 8 \\
\hline Injeção & 431827,2 & 431827,2 & 345461,8 & 431827,2 & 345461,8 & 431827,2 & 431827,2 & 431827,2 \\
\hline Lixamento & 301593,6 & 301593,6 & 241274,9 & 301593,6 & 241274,9 & 301593,6 & 301593,6 & 301593,6 \\
\hline
\end{tabular}

Fonte: Autores (2020)

A partir dos dados gerados pelo PMP (Quadro 16) e dos perfis de recursos de cada modelo (Quadro 17), obteve-se os parâmetros para o cálculo subsequente.

Quadro 16 - Dados do PMP de todos os produtos

\begin{tabular}{|c|c|c|c|c|c|c|c|c|}
\hline \multirow{2}{*}{ PMP } & \multicolumn{7}{|c|}{ PERÍODO } \\
\cline { 2 - 9 } & SEM 1 & SEM 2 & SEM 3 & SEM 4 & SEM 5 & SEM 6 & SEM 7 & SEM 8 \\
\hline M40210 & 4047 & 3973 & 2969 & 3629 & 2440 & 2396 & 1791 & 2188 \\
\hline M40328 & 3646 & 3579 & 2675 & 3269 & 2198 & 2158 & 1613 & 1971 \\
\hline M40263 & 1759 & 1726 & 1290 & 1577 & 1061 & 1041 & 778 & 951 \\
\hline M40305 & 3320 & 3259 & 2436 & 2977 & 2002 & 1965 & 1469 & 1795 \\
\hline M40262 & 3269 & 3209 & 2398 & 2931 & 1971 & 1935 & 1446 & 1767 \\
\hline
\end{tabular}

Fonte: Autores (2020) 
Quadro 17 - Perfil de recurso para os modelos

\begin{tabular}{|c|c|c|}
\hline \multicolumn{3}{|c|}{ PERFIL DE RECURSOS } \\
\hline \multirow{2}{*}{ MODELO } & \multicolumn{2}{|c|}{ TEMPO UNITÁRIO (s) } \\
\cline { 2 - 3 } & INJEÇÃO & LIXAMENTO \\
\hline 40210 & 27,5 & 14,4 \\
\hline 40305 & 21,6 & 0 \\
\hline 40328 & 27,5 & 0 \\
\hline 40262 & 25 & 0 \\
\hline 40263 & 30 & 0 \\
\hline
\end{tabular}

Fonte: Autores (2020)

A quantidade de segundos necessários semanais para a produção dos cincos itens em cada setor, obtidos através da multiplicação do tempo de produção dos itens em cada centro de trabalho pela demanda correspondente a estes é mostrado no Quadro 18.

Quadro 18 - Quantidade de segundos necessários por semana por setor

\begin{tabular}{|c|c|c|c|c|c|c|c|c|}
\hline \multicolumn{7}{|c|}{ SEGUNDOS NECESSÁRIOS } \\
\hline $\begin{array}{c}\text { CENTRO DE } \\
\text { TRABALHO }\end{array}$ & SEM 1 & SEM 2 & SEM 3 & SEM 4 & SEM 5 & SEM 6 & SEM 7 & SEM 8 \\
\hline INJEÇÃO & 417764,5 & 410079,4 & 306477,6 & 374583,2 & 251893,2 & 247284 & 184830,4 & 225849,5 \\
\hline LIXAMENTO & 58276,8 & 57211,2 & 42753,6 & 52257,6 & 35136 & 34502,4 & 25790,4 & 31507,2 \\
\hline
\end{tabular}

Fonte: Autores (2020)

Diante dos resultados alcançados, tornou-se possível calcular a utilização da capacidade por meio da divisão dos segundos necessários pelos disponíveis nos dois setores, como indica o Quadro 19.

Quadro 19 - Cálculo de utilização semanal nos setores de Injeção e Lixamento

\begin{tabular}{|c|c|c|c|c|c|c|c|c|}
\hline \multicolumn{7}{|c|}{ UTILIZAÇÃO DA CAPACIDADE } \\
\hline $\begin{array}{c}\text { CENTRO DE } \\
\text { TRABALHO }\end{array}$ & SEM 1 & SEM 2 & SEM 3 & SEM 4 & SEM 5 & SEM 6 & SEM 7 & SEM 8 \\
\hline INJEÇÃO & $97 \%$ & $95 \%$ & $89 \%$ & $87 \%$ & $73 \%$ & $57 \%$ & $43 \%$ & $52 \%$ \\
\hline LIXAMENTO & $13 \%$ & $13 \%$ & $12 \%$ & $12 \%$ & $10 \%$ & $8 \%$ & $6 \%$ & $7 \%$ \\
\hline
\end{tabular}

Fonte: Autores (2020)

Os resultados obtidos mostram que a utilização para os setores de Injeção e Lixamento no PMP atendem à demanda de produção solicitada com excedente na capacidade, 


\subsection{Elaboração do MRP I}

Para iniciar o MRP I, foi necessário a definição da proporção de ABS para os produtos estudados. O Quadro 20 apresenta a árvores dos produtos com a necessidade da matéria-prima em kg.

Quadro 20 - Árvores dos modelos

\begin{tabular}{|c|c|c|}
\hline \multicolumn{3}{|c|}{ ÁRVORE DO PRODUTO } \\
\hline \multirow{2}{*}{ MODELO } & \multicolumn{2}{|c|}{ PROPORÇÃO } \\
\cline { 2 - 3 } & QUANTIDADE (und) & ABS (kg) \\
\hline 40210 & 1 & 0,157 \\
\hline 40305 & 1 & 0,124 \\
\hline 40328 & 1 & 0,115 \\
\hline 40262 & 1 & 0,092 \\
\hline 40263 & 1 & 0,169 \\
\hline
\end{tabular}

Fonte: Autores (2020)

Em seguida, realizou-se os cálculos das quantidades requeridas para o cumprimento do planejamento semanal definido no PMP. Através das informações coletadas considerou-se o lead time de ressuprimento de 2 semanas, a quantidade de estoque de ABS disponível na organização como 3192 kg e o lote econômico de compra sendo 840 kg.

Após o estabelecimento desses parâmetros, realizou-se a elaboração do registro básico do MRP (Quadro 21).

Quadro 21 - MRP para o mix de produtos

\begin{tabular}{|c|c|c|c|c|c|c|c|c|c|}
\cline { 2 - 10 } \multicolumn{1}{c|}{} & \multicolumn{9}{c|}{ MATÉRIA PRIMA: ABS } \\
\hline SEMANA & 0 & 1 & 2 & 3 & 4 & 5 & 6 & 7 & 8 \\
\hline NECESSIDADE BRUTA & & 2064,368 & 2026,384 & 1514,448 & 1851,001 & 1244,739 & 1221,951 & 913,352 & 1116,044 \\
\hline ESTOQUE DISPONÍVEL & & 3192 & 1127,632 & 781,248 & 106,8 & 775,799 & 371,06 & 829,109 & 755,757 \\
\hline RECEBIMENTO PROGRAMADO & & & 1680 & 840 & 2520 & 840 & 1680 & 840 & 0 \\
\hline NECESSIDADE LÍQUIDA & & 0 & 0 & 0 & 0 & 0 & 0 & 0 & 0 \\
\hline LIBERAÇÃO DE ORDEM & 1680 & 840 & 2520 & 840 & 1680 & 840 & & \\
\hline
\end{tabular}

Fonte: Autores (2020)

Durante o planejamento, tornou-se indispensável desde a primeira semana a reposição dos estoques de $\mathrm{ABS}$, sendo necessário ordens de pedido até a semana cinco.

\subsection{Elaboração do SFC}

Inicialmente aplicou-se a regra do menor tempo de processamento no setor de injeção para a definição da ordem de manufatura dos produtos, onde foi utilizado os tempos de operação em 
cada centro de trabalho. Este tipo de sequenciamento foi escolhido por agilizar o carregamento para o setor seguinte, pois o produto M40210 precisa de lixamento, de modo que o centro de trabalho fica ocioso até seu processamento. A sequência determinada é expressa no Quadro 22.

Quadro 22 - Regra do menor tempo de processamento

\begin{tabular}{|c|c|c|}
\cline { 2 - 3 } \multicolumn{1}{c|}{} & MENOR TEMPO DE PROCESSAMENTO - INJEÇÃO \\
\cline { 2 - 3 } \multicolumn{1}{c|}{} & INJEEÇÃO & LIXAMENTO \\
\hline M40305 & 21,6 & 0 \\
\hline M40262 & 25 & 0 \\
\hline M40210 & 27,5 & 14,4 \\
\hline M40328 & 27,5 & 0 \\
\hline M40263 & 30 & 0 \\
\hline
\end{tabular}

Fonte: Autores (2020)

Em seguida, para exemplificar o planejamento de um sequenciamento foi escolhida a semana que apresentou a maior capacidade de utilização no PMP, para assim determinar os dias em que cada produto deveria ser fabricado, presumindo que os itens somente podem continuar o fluxo produtivo após a finalização do lote na injeção. Vale ressaltar que o setor de lixamento opera apenas em um turno, enquanto a injeção trabalha durante dois turnos. Assim, foi considerado as 7,98 horas disponíveis de trabalho no setor de injeção e 4,18 horas no setor de lixamento, onde este tempo foi determinado através da quantidade de turnos por operação, o tempo direcionado à produção do mix de produto deste estudo e uma eficiência de $85 \%$. O Quadro 23 mostra a produção diária dos produtos para a semana 1.

Quadro 23 - Sequenciamento da produção para semana 1

\begin{tabular}{|c|c|c|c|c|c|c|c|c|c|c|}
\hline & \multicolumn{10}{|c|}{ SEMANA I } \\
\hline & \multicolumn{2}{|c|}{$01 / a b r$} & \multicolumn{2}{|c|}{$02 / a b r$} & \multicolumn{2}{|c|}{$03 / a b r$} & \multicolumn{2}{|c|}{$04 / a b r$} & \multicolumn{2}{|c|}{$05 / a b r$} \\
\hline & \multicolumn{10}{|c|}{ INJEEÇÃO } \\
\hline & HORAS & QUANT & HORAS & QUANT & HORAS & QUANT & HORAS & QUANT & HORAS & QUANT \\
\hline M40305 & 7,980 & 3990 & 0,114 & 57 & & & & & & \\
\hline M40262 & & & 7,866 & 3398 & 0,574 & 248 & & & & \\
\hline M40210 & & & & & 4,479 & 1759 & & & & \\
\hline M40328 & & & & & 2,921 & 1147 & 5,533 & 2173 & & \\
\hline M40263 & & & & & & & 2,444 & 880 & 6,636 & 2389 \\
\hline \multirow[t]{3}{*}{ TOTAL } & 7,980 & & 7,980 & & 7,974 & & 7,978 & & 6,636 & \\
\hline & \multicolumn{10}{|c|}{ LIXAMENTO } \\
\hline & HORAS & QUANT & HORAS & QUANT & HORAS & QUANT & HORAS & QUANT & HORAS & QUANT \\
\hline \multicolumn{11}{|l|}{ M40305 } \\
\hline \multicolumn{11}{|l|}{ M40262 } \\
\hline M40210 & & & & & & & & & 3,359 & 1759 \\
\hline \multicolumn{11}{|l|}{ M40328 } \\
\hline \multicolumn{11}{|l|}{ M40263 } \\
\hline TOTAL & & & & & & & & & & \\
\hline
\end{tabular}

Fonte: Autores (2020) 


\section{Considerações finais}

O estudo em questão buscou planejar as atividades de fabricação dos principais produtos em uma empresa do ramo de injetáveis na cidade de Russas - Ceará. A programação das quantidades e o período indicado para a manufatura de saltos foi encontrada através da aplicação do MRP II, fazendo com que o estudo alcançasse objetivos previamente estabelecidos.

Com esta aplicação, foi constatado a importância e o impacto deste modelo para o gerenciamento da produção nas organizações, de modo que proporcionem a garantia da competitividade dentro do mercado, assegurando maiores benefícios à empresa, planejando sua produção da melhor forma possível de modo a atender e satisfazer seus clientes. Entre as dificuldades encontradas destaca-se as características dos dados utilizados, pois estes dificultaram a interpretação e definição do método indicado para realização da previsão de demanda a ser usada no PVO.

De acordo com os objetivos do Planejamento e Controle da Produção, é necessário o acompanhamento e controle das atividades proposta neste estudo de caso. Portanto, para a validação e contínua melhoria dos processos da empresa estudada, além de uma maior agregação de valor, propõe-se o aprofundamento das ferramentas, sua usabilidade e aplicabilidade dentro da organização.

\section{REFERÊNCIAS}

ABICALÇADOS. Relatório setorial indústria de calçados Brasil 2018. Disponível em: <https://drive.google.com/file/d/18atEww9qvlQeMu3EutWURtHdTcXFNCnQ/view> Acesso em: 07 de abril de 2019.

BERTAGLIA, Paulo Roberto. Logística e gerenciamento da cadeia de abastecimento. 2. Ed. São Paulo: Saraiva, 2009.

BONNEY, Maurice. Reflections on production planning and control (PCP). Revista Gestão \& Produção. Vol. 7, número 3, p. 181-207, 2000.

CORRÊA, Henrique L.; CORRÊA, Carlos A. Administração de produção e operações: manufatura e serviços: uma abordagem estratégica. 3 ed. São Paulo: Atlas, 2012.

CORRÊA, Henrique L.; GIANESI, Irineu G. N. JIT, MRP II e OPT: um enfoque estratégico. São Paulo: Atlas, 2002. 
ESTENDER, Antonio Carlos; AEQUEIRA, Gisela Romariz; SIQUEIRA, Nilza Aparecida dos Santos; CANDIDO, Guilherme Junqueira. A Importância do Planejamento e Controle de Produção. Anais do VI SINGEP. São Paulo: SP, 2017.

GIL, A. C. Como elaborar projetos de pesquisa. 4 ed. São Paulo: Atlas, 2002.

LUSTOSA, Leonardo Junqueira; MESQUITA, Marco Aurélio de; QUELHAS, Osvaldo Luiz Gonçalves; OLIVEIRA, Rodrigo Jorge de. Planejamento e controle da produção. Rio de Janeiro: Elsevier, 2008.

MARTINS, Petrônio G.; LAUGENI, Fernando Piero. Administração da produção fácil. São Paulo: Saraiva, 2012.

OLHAGER, J.; RUDBERG, M.; WIKNER, J. Long-term capacity management: Linking the perspectives from manufacturing strategy and sales and operations planning. International Journal of Production Economics, v. 69, p. 215-225, 2001.

PEINADO, Jurandir; GRAEML, Alexandre Reis. Administração da produção: operações industriais e de serviços. Curitiba: UnicenP, 2007.

ROMANZINI, Fernanda; RIBEIRO, José Luis Duarte. Uma proposta de planejamento de produção vinculada a margem de lucro dos produtos manufaturados. Revista Produção Online, v. 17, n. 1, p. 200-221, 2017.

SILVA, Airton Marques da. Metodologia da Pesquisa. 2 ed. Fortaleza: EdUECE, 2015.

SILVA, Luciano Santos da. Planejamento dos Recursos de Manufatura - MRP II. Centro Universitário Leonardo da Vinci, 2012.

SLACK, Nigel; CHAMBERS, Stuart; JOHNSTON, Robert. Administração da produção. 3. ed. São Paulo: Atlas, 2009.

SLACK, Nigel; CHAMBERS, Stuart; HARLAND, Christine; HARRISON, Alan; JOHNSTON, Robert. Administração da produção. 1. ed. São Paulo: Atlas, 2006.

VENANZI, Délvio; SILVA, Orlando Roque da. Gerenciamento da produção e operações. 1 ed. Rio de Janeiro: LTC, 2013. 


\section{ANEXOS}

ANEXO I - Fluxograma do processo produtivo

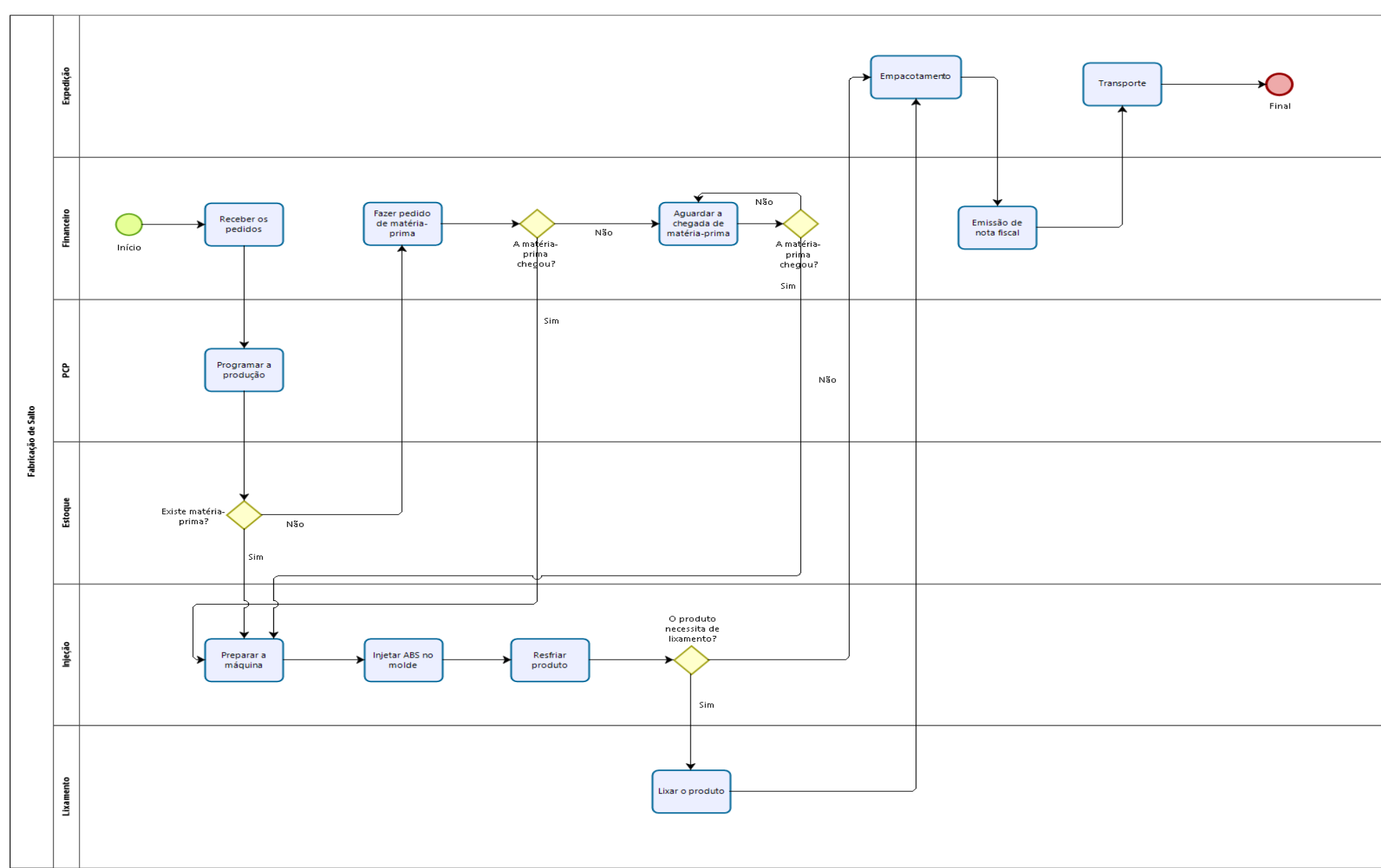


ANEXO II - Cálculo dos fatores globais

\begin{tabular}{|c|c|c|c|c|c|c|c|c|c|c|c|}
\hline \multicolumn{10}{|c|}{ CÁLCULO DOS FATORES GLOBAIS DE UTILIZAÇÃO } \\
\hline $\begin{array}{c}\text { CENTRO DE } \\
\text { TRABALHO }\end{array}$ & M40210 & M40262 & M40263 & M40305 & M40328 & $\begin{array}{c}\text { VOL. } \\
\text { M40210 } \\
(\%)\end{array}$ & $\begin{array}{c}\text { VOL. } \\
\text { M40262 } \\
(\%)\end{array}$ & $\begin{array}{c}\text { VOL. } \\
\text { M40263 } \\
(\%)\end{array}$ & $\begin{array}{c}\text { VOL. } \\
\text { M40305 } \\
(\%)\end{array}$ & $\begin{array}{c}\text { VOL. } \\
\text { M40328 } \\
(\%)\end{array}$ & $\begin{array}{c}\text { FATOR } \\
\text { GLOBAL }\end{array}$ \\
\hline INJEÇÃO & 27,5 & 25 & 30 & 21,6 & 27,5 & $25,3 \%$ & $20,6 \%$ & $10,7 \%$ & $20,3 \%$ & $23,1 \%$ & 26,1 \\
\hline LIXAMENTO & 14,4 & 0 & 0 & 0 & 0 & $25,3 \%$ & $20,6 \%$ & $10,7 \%$ & $20,3 \%$ & $23,1 \%$ & 3,6 \\
\hline
\end{tabular}

